\title{
Exploring of the molecular mechanism of rhinitis via bioinformatics methods
}

\author{
YUFEN SONG and ZHAOHUI YAN \\ Department of Otolaryngology, The Third Central Hospital of Tianjin, Tianjin 300170, P.R. China
}

Received March 22, 2017; Accepted October 6, 2017

DOI: $10.3892 / \mathrm{mmr} .2017 .8213$

\begin{abstract}
The aim of this study was to analyze gene expression profiles for exploring the function and regulatory network of differentially expressed genes (DEGs) in pathogenesis of rhinitis by a bioinformatics method. The gene expression profile of GSE43523 was downloaded from the Gene Expression Omnibus database. The dataset contained 7 seasonal allergic rhinitis samples and 5 non-allergic normal samples. DEGs between rhinitis samples and normal samples were identified via the limma package of $\mathrm{R}$. The webGestal database was used to identify enriched Kyoto Encyclopedia of Genes and Genomes (KEGG) pathways of the DEGs. The differentially co-expressed pairs of the DEGs were identified via the DCGL package in $\mathrm{R}$, and the differential co-expression network was constructed based on these pairs. A protein-protein interaction (PPI) network of the DEGs was constructed based on the Search Tool for the Retrieval of Interacting Genes database. A total of 263 DEGs were identified in rhinitis samples compared with normal samples, including 125 downregulated ones and 138 upregulated ones. The DEGs were enriched in 7 KEGG pathways. 308 differential co-expression gene pairs were obtained. A differential co-expression network was constructed, containing 212 nodes. In total, 148 PPI pairs of the DEGs were identified, and a PPI network was constructed based on these pairs. Bioinformatics methods could help us identify significant genes and pathways related to the pathogenesis of rhinitis. Steroid biosynthesis pathway and metabolic pathways might play important roles in the development of allergic rhinitis (AR). Genes such as CDC42 effector protein 5, solute carrier family 39 member A11 and PR/SET domain 10 might be also associated with the pathogenesis of AR, which provided references for the molecular mechanisms of AR.
\end{abstract}

Correspondence to: Dr Yufen Song, Department of Otolaryngology, The Third Central Hospital of Tianjin, 83 Jin-Tang Road, He Dong, Tianjin 300170, P.R. China

E-mail: yfsongnice@outlook.com

Key words: allergic rhinitis, Gene Expression Omnibus database, bioinformatics, differentially expressed genes

\section{Introduction}

Allergic rhinitis (AR) is a symptomatic disorder of the nose induced after exposure to allergens via IgE-mediated hypersensitivity reactions, which are characterized by the cardinal symptoms of watery rhinorrhea, nasal obstruction, nasal itching and sneezing (1). It represents one of the greatest health problems in modern societies (2). A conservative estimate revealed that AR occurs in over 500 million people around the world (3). The prevalence is about $10-30 \%$ in adults and nearly $40 \%$ in children (4). This makes AR become one of the most common allergic diseases in the world, with increasing prevalence and often far-reaching consequences for quality of life. Allergen-specific immunotherapy (SIT) is the most available treatment for AR. It can alter the natural course of allergic disease by preventing new sensitization/onset and providing long-term remission after discontinuation of treatment (5). However, the conventional SIT, subcutaneous injection, requires frequent hospital visits and is painful, resulting in a low patient compliance. Furthermore, it may cause some adverse events such as anaphylaxis (6). It is urgent to make a deeper understanding of the pathogenesis of AR and find new therapeutic methods.

Recent studies showed that genetic factors played important roles in the development of AR. There was ample evidence suggesting that AR was a complex multifactorial disorder including both genetic and environmental factors $(7,8)$. Several genes and pathways had been reported to be associated with AR. One of the important biological signals involved in the pathogenesis of AR was histamine, which was released after relevant antigenic stimulation of sensitized subjects, initiating the early phase of allergic reaction (9). Moreover, one research demonstrated that thymic stromal lymphopoietin (TSLP) gene SNP rs1837253 was associated with reduced odds for AR in boys with asthma (10). In addition, apolipoprotein A-IV was also reported to be associated with the pathogenesis of AR, and could be served as a candidate target molecule for the treatment of seasonal AR (3). However, the precise molecular mechanism of AR is still not well understood.

In this study, differentially expressed genes (DEGs) in AR samples compared with normal samples were identified through bioinformatics methods. The construction of the differential co-expression network and the protein-protein interaction (PPI) network might provide us a better understanding of the pathogenesis of AR. Our study might provide references for the diagnosis and therapy of AR. 


\section{Materials and methods}

Microarray data. The gene expression profile of GSE43523 was downloaded from National Center of Biotechnology Information (NCBI) Gene Expression Omnibus (GEO; http://www.ncbi.nlm.nih.gov/geo/) database. The dataset contained 7 nasal epithelial cells of seasonal AR samples and 5 nasal epithelial cells of non-allergic normal samples. The expression profile was detected based on GPL6883 Illumina HumanRef-8 v3.0 expression beadchip platform.

Data processing and identification of DEGs. The raw data were background corrected, quantile normalized and $\log 2$ transformed using the preprocessCore package in $\mathrm{R}$ (11). Affymetrix probe IDs were converted to official gene symbol. If multiple probes corresponded to one given gene, the mean expression value of those probes was defined as the gene expression value. DEGs in rhinitis samples compared with normal samples were identified via the limma package of $\mathrm{R}$ (12). Bonferroni and Hochberg method was used for the correction of P-value. The threshold was $\mathrm{P}<0.05$ and $\mid \log 2$ (fold change) $>$ 0.58 . The threshold was $\mid \log 2$ (fold change) $\mid>0.58$ that mean gene expression quantity in rhinitis samples change $>1.5$-fold compared with normal samples. Besides, hierarchical clustering analysis of rhinitis samples and normal samples based on the DEGs were performed.

Pathway enrichment analysis. To further explore the biological functions and involved pathways of the DEGs, Kyoto Encyclopedia of Genes and Genomes (KEGG) pathway enrichment analysis was performed based on the webGestal database with the threshold of $\mathrm{P}<0.05$ (13).

Construction of the differential co-expression network. DCGL was an $\mathrm{R}$ package for identifying differentially co-expressed genes and links from gene expression microarray data (14). It could examine the expression correlation based on the exact co-expression changes of gene pairs between two conditions (15). In this study, the co-expression values for each pair of the DEGs in rhinitis samples and normal samples were calculated via the DCGL package in R. Gene pairs with different signs of co-expression values in two types of samples were selected. Then, the differential co-expression pairs were identified according to the criterion: Absolute value of the difference of co-expression values in two samples $>1.5$. The differential co-expression network of the DEGs was constructed based on these pairs.

Construction of the PPI network. Search Tool for the Retrieval of Interacting Genes (STRING) (http://string-db.org/) (16) was an online database for predicting functional interactions between proteins (17). In this study, PPI pairs of the DEGs were selected based on the STRING database with the threshold of combined score $>0.4$. The PPI network of the DEGs was constructed based on these pairs.

\section{Results}

The DEGs. A total of 263 DEGs were identified in rhinitis samples compared with normal samples, including 125 downregulated ones and 138 upregulated ones (Fig. 1). The top 20
Table I. The top 20 DEGs in AR samples compared with normal samples.

\begin{tabular}{llr}
\hline Gene name & P-value & LogFC \\
\hline ST3GAL5 & $0.83 \times 10^{-4}$ & -0.655 \\
NR1D2 & $1.04 \times 10^{-4}$ & -1.144 \\
AKR1B1 & $1.46 \times 10^{-4}$ & -1.255 \\
HIST1H2BD & $1.58 \times 10^{-4}$ & 0.985 \\
TMEM125 & $1.72 \times 10^{-4}$ & 0.698 \\
MAP3K2 & $2.38 \times 10^{-4}$ & -1.170 \\
AGR2 & $3.98 \times 10^{-4}$ & 0.927 \\
RNF217 & $6.40 \times 10^{-4}$ & -1.171 \\
CST1 & $8.47 \times 10^{-4}$ & 6.013 \\
LIN54 & $8.74 \times 10^{-4}$ & -0.913 \\
ZNF750 & $9.18 \times 10^{-4}$ & -1.049 \\
DHCR24 & $9.70 \times 10^{-4}$ & 0.711 \\
SLC39A11 & $9.82 \times 10^{-4}$ & 0.669 \\
ATP2C2 & $10.88 \times 10^{-4}$ & 1.218 \\
CAMK2G & $10.93 \times 10^{-4}$ & -1.003 \\
CLC & $11.26 \times 10^{-4}$ & 2.565 \\
SDPR & $11.28 \times 10^{-4}$ & 1.025 \\
IL20RB & $11.74 \times 10^{-4}$ & 1.237 \\
FAM46B & $13.36 \times 10^{-4}$ & 0.860 \\
ANKRD13C & $13.44 \times 10^{-4}$ & -0.906 \\
\hline
\end{tabular}

DEGs, differentially expressed genes; AR, allergic rhinitis FC, fold change.

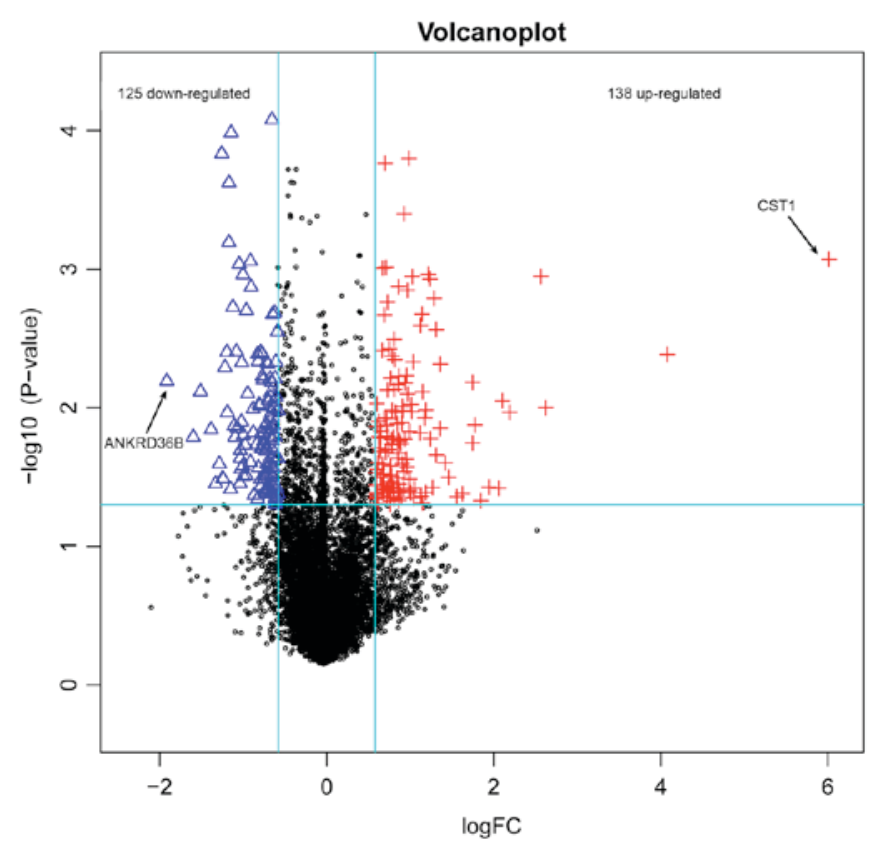

Figure 1 . The differentially expressed genes in allergic rhinitis samples compared with normal samples. FC, fold change.

DEGs according to P-value were listed in Table I. Clustering analysis revealed a clearly distinct expression of all DEGs between rhinitis samples and normal samples (Fig. 2). From 


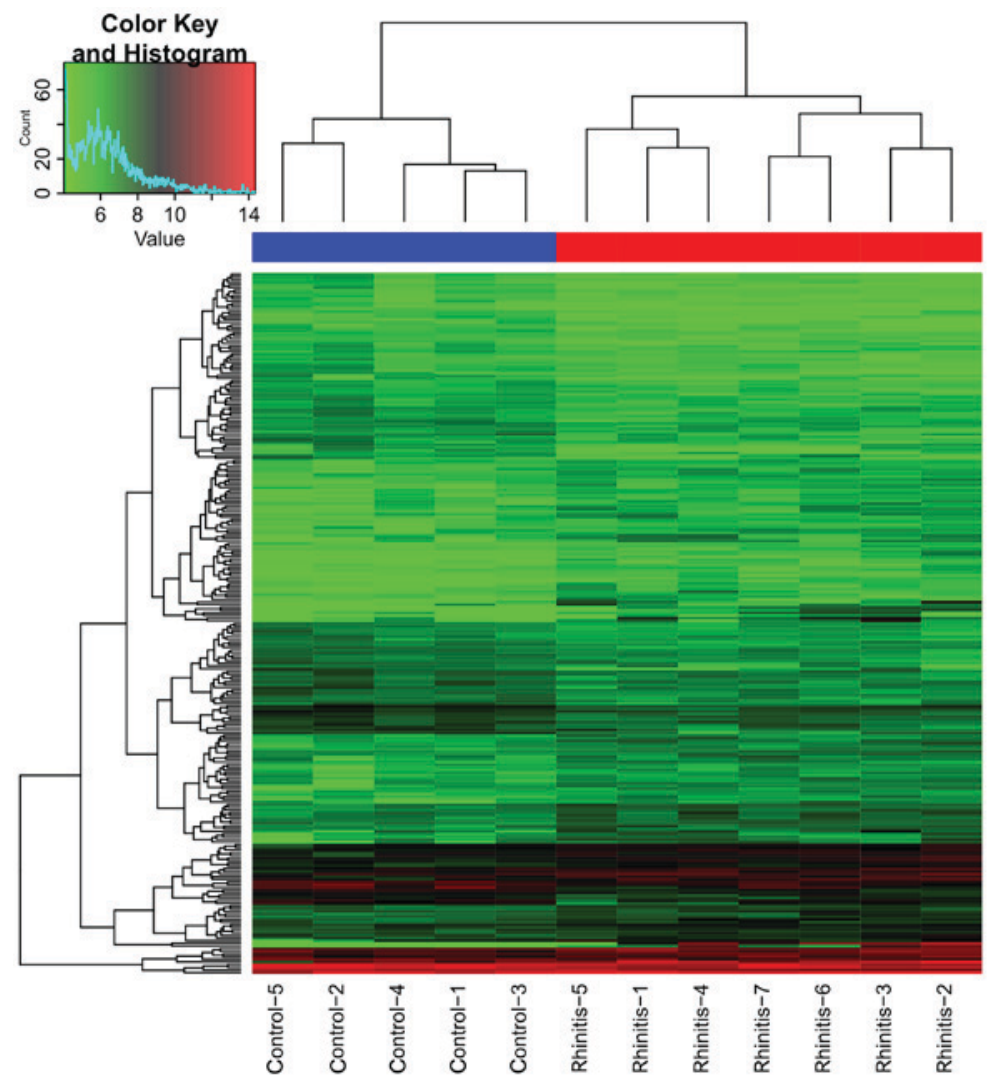

Figure 2. The hierarchical cluster analysis of the allergic rhinitis samples and normal samples based on the differentially expressed genes. Green, black and red colors represent the expression values of the DEGs, as indicated by the histogram. DEGs, differentially expressed genes.

the heatmap (Fig. 2), we found that the gene expression of 5 nasal epithelial cells of non-allergic normal samples was distinguished from the 7 nasal epithelial cells of seasonal AR samples.

The enriched pathways. Seven enriched KEGG pathways of the DEGs were obtained in the webGestal database (Table II). The top 4 pathways were fructose and mannose metabolism, riboflavin metabolism, renin-angiotensin system (RAS), amino sugar and nucleotide sugar metabolism respectively.

The differential co-expression network. A total of 308 differential co-expression gene pairs were obtained. The co-expression network based on these pairs was constructed (Fig. 3), which contained 212 nodes. The top 20 nodes according to the degree were listed in Table III.

The PPI network. 148 PPI pairs were identified by the STRING database. A PPI network was constructed based on these pairs and contained 125 genes (Fig. 4). The top 20 nodes of the PPI network were listed in Table IV.

\section{Discussion}

AR significantly affects the quality of the patient's daily life. Despite the development of various of pharmacological methods, avoidance of the allergen is usually not possible and symptom relief is often limited (18). The precise pathogenesis of AR is still not well understood. In this study, DEGs in AR samples compared with normal samples were identified via
Table II. The enriched KEGG pathways of the DEGs.

\begin{tabular}{|c|c|c|}
\hline Category & Pathway name & P-value \\
\hline KEGG pathway & $\begin{array}{l}\text { Fructose and mannose } \\
\text { metabolism }\end{array}$ & 0.001 \\
\hline KEGG pathway & Riboflavin metabolism & 0.008 \\
\hline KEGG pathway & Renin-angiotensin system & 0.020 \\
\hline KEGG pathway & $\begin{array}{l}\text { Amino sugar and nucleotide } \\
\text { sugar metabolism }\end{array}$ & 0.027 \\
\hline KEGG pathway & Steroid biosynthesis & 0.024 \\
\hline KEGG pathway & Ribosome & 0.027 \\
\hline KEGG pathway & Metabolic pathways & 0.032 \\
\hline
\end{tabular}

KEGG, Kyoto Encyclopedia of Genes and Genomes; DEGs, differentially expressed genes.

bioinformatics methods. Then the DEGs were further analyzed by the construction of differential co-expression network and PPI network to a better understanding of the molecular mechanism of AR.

The gene expression profile of GSE43523 contained 7 nasal epithelial cells of seasonal AR samples and 5 nasal epithelial cells of non-allergic normal samples. Small sample size was a limitation of the study but the results of our analysis was reliable to a certain extent. The small samples of studies have been recognized by many researchers. For example, Zhu et al identified endometrial cancer prognosis markers which the 
Table III. The top 20 nodes in the differential co-expression network with high degree.

\begin{tabular}{lc}
\hline Gene & Degree \\
\hline CDC42EP5 & 14 \\
SERPINF1 & 13 \\
SLC39A11 & 13 \\
SLC7A1 & 12 \\
MAGEE1 & 10 \\
FABP6 & 9 \\
ABCA1 & 8 \\
TRNP1 & 8 \\
C1orf112 & 7 \\
DNAJB9 & 7 \\
GCNT3 & 7 \\
GOLT1A & 7 \\
HLA & 7 \\
MRPL52 & 7 \\
NR2C2 & 7 \\
NT5DC2 & 7 \\
POLD4 & 7 \\
AKR1B1 & 7 \\
CHST6 & 7 \\
CLDN1 & 7 \\
\hline
\end{tabular}

tissue samples for the microarray study consisted of 7 control samples, 3-G1 samples, 8-G2 samples and 2-G3 samples (19). A total of 263 DEGs were identified in rhinitis samples compared with normal samples which were further analyzed by the construction of differential co-expression network and PPI network to a better understanding of the molecular mechanism of AR. Individual analysis of the 263 DEGs was not performed as this was not considered necessary.

The enriched KEGG pathways of the DEGs were fructose and mannose metabolism, riboflavin metabolism, RAS, amino sugar and nucleotide sugar metabolism, steroid biosynthesis, ribosome and metabolic pathways. Many of these pathways were associated with the pathogenesis of AR. Angiotensin was a peptide hormone that caused vasoconstriction and a subsequent increase in blood pressure. It was part of the RAS, which was a major target for drugs that lowered blood pressure (20). Angiotensin converting enzyme (ACE), which contained 26 exons and 25 introns, was reported to be an important AR susceptibility gene (21). ACE was essential in converting angiotensin I into angiotensin II, which was an mainly effector molecule in the RAS and acted as pro-inflammatory modulator in the augmentation of immune responses $(22,23)$. The insertion or deletion polymorphism of ACE was a risk factor for AR and verified by large number and representative population (24). A steroid was an organic compound with four rings arranged in a specific configuration (25). Steroid were generally considered the most effective medications for the management of inflammatory diseases including asthma and AR (26). The corticosteroids could be given locally into the nose and bronchi without risk of systemic adverse effects. The introduction of potent corticosteroids in the treatment of AR
Table IV. The top 20 nodes in the PPI network with high degree.

\begin{tabular}{lr}
\hline Gene & Degree \\
\hline PRDM10 & 22 \\
EP300 & 12 \\
ITGA2 & 11 \\
RRAS & 7 \\
VASN & 6 \\
ATP12A & 5 \\
SERPINE2 & 5 \\
SRSF7 & 5 \\
ABCA1 & 4 \\
ALDH3A1 & 4 \\
ASF1A & 4 \\
EDF1 & 4 \\
FKBP4 & 4 \\
GZMB & 4 \\
HPS3 & 4 \\
MAF & 4 \\
MUC2 & 4 \\
SQSTM1 & 4 \\
TYRO3 & 4 \\
ALDH16A1 & 4 \\
\end{tabular}

PPI, protein-protein interaction.

had been a major therapeutic advance, and had emphasized the importance of pharmacological and morphological aspects of AR (27). Topical steroid treatment of AR could decrease nasal fluid TH2 cytokines, eosinophils, eosinophil cationic protein and IgE (28). Metabolic pathways also played critical roles in the development of AR. Kinin metabolism in human nasal secretions during experimentally could induce AR (29). In addition, one study demonstrated that serum tryptophan metabolism could be served as a biomarker in patients with AR (30). The metabolism of vitamin D was also reported to be different in AR patients (31).

The top 5 genes in the differential co-expression network according to the degree were CDC42EP5, SERPINF1, SLC39A11, SLC7A1 and MAGEE1, respectively. While the top 5 genes in the PPI network were PRDM10, EP300, $I T G A 2, R R A S$ and $V A S N$, respectively. Shi and his team found that FOS, JUN, and CEBPD may play crucial roles during the process of seasonal allergic rhinitis (SAR) by the microarray data GSE50101 (32). The different results with our study may be caused by distinction between AR and SAR. AR was an inflammatory diseases, and many of the above genes were reported to be associated with the pathogenesis of AR or inflammatory diseases (33). SLC39A11 encoded a type of human zinc transporter, which was one of the critical regulators that maintained intracellular zinc concentrations, and played a role in regulating cell survival during inflammation $(34,35)$. Zinc was an essential micronutrient and cytoprotectant involved in the host response to inflammatory stress. Zip protein was demonstrated to be 


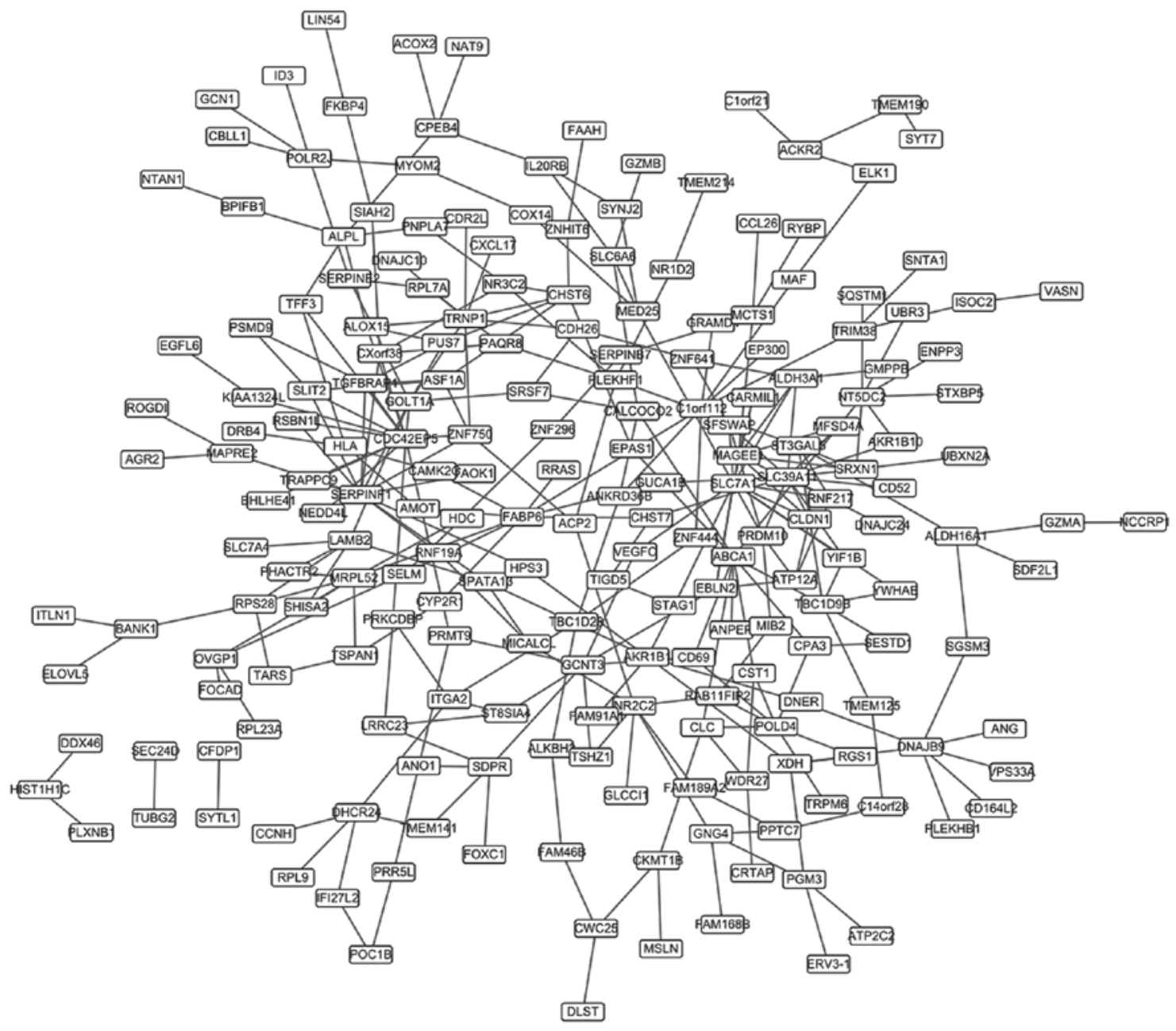

Figure 3. The differential co-expression network of the differentially expressed genes.

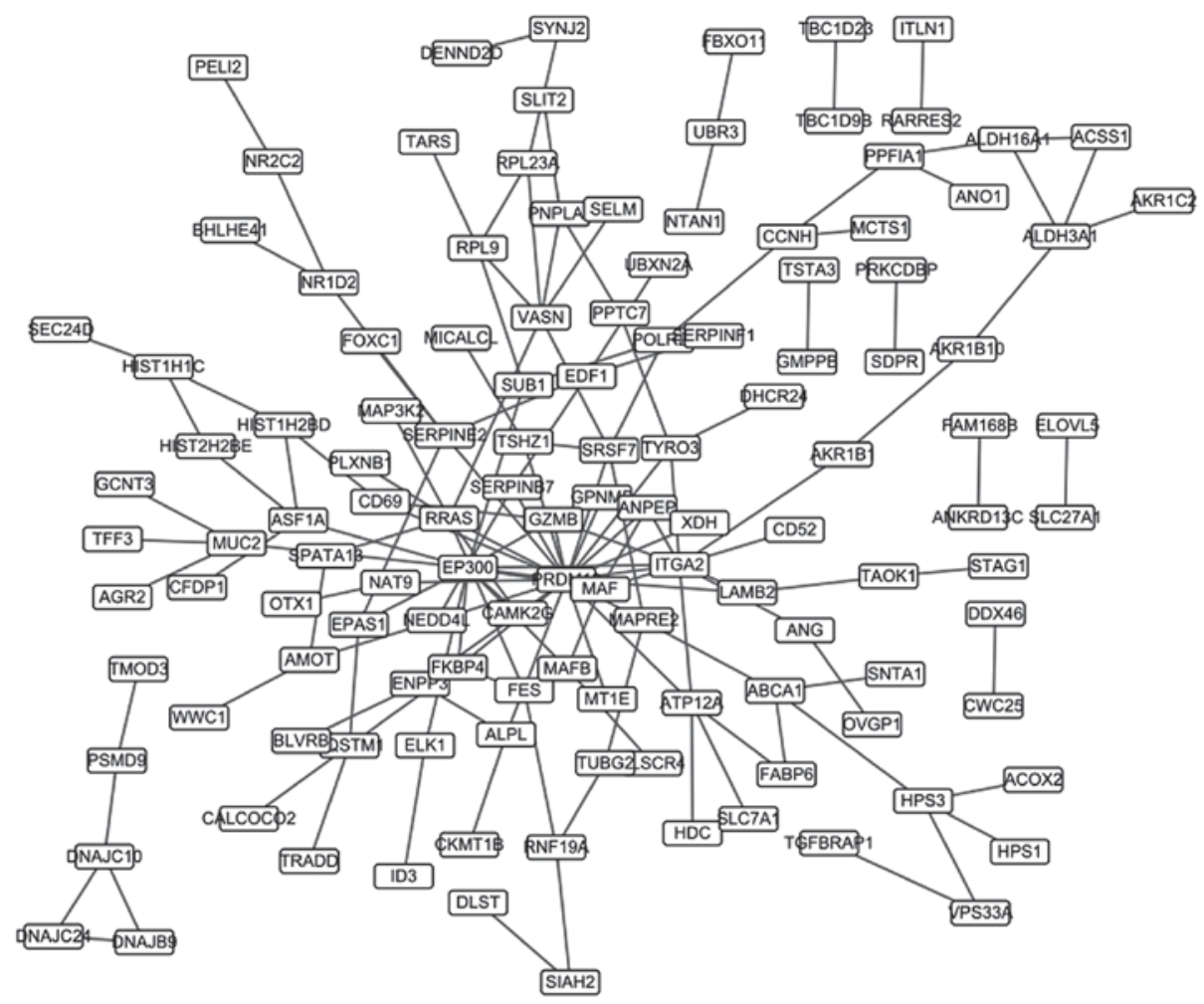

Figure 4. The protein-protein interaction network of the differentially expressed genes. 
an essential zinc importer at the onset of inflammation for facilitating cytoprotection (36). Zinc was also an antioxidant and had anti-inflammatory actions. Zinc could induce A20 which inhibited nuclear transcription factor $\kappa \mathrm{B}(\mathrm{NF}-\kappa \mathrm{B})$ activation resulting in decreased generation of inflammatory cytokines (37). Zinc deficiency was confirmed in patients with AR (38). CDC42EP5 was a member of CDC42 effector protein family. It could bind to $\mathrm{CDC} 42$ and regulate its function negatively (39). CDC42 was a Rho-family GTPase. It had been implicated in several signal transduction pathways, including $\mathrm{NF}-\kappa \mathrm{B}$ activation, activation of the c-Jun N-terminal MAP kinase and stimulation of the NF- $\kappa \mathrm{B}(40,41)$. CDC42 could be activated by the inflammatory cytokines TNF $\alpha$ and IL-1, which was associated with the development of inflammatory diseases (42). In addition, CDC42 signaling was identified as a mediator of chronic inflammation associated with endothelial senescence. Inhibition of CDC42 or NF- $\kappa \mathrm{B}$ signaling would attenuate the sustained upregulation of pro-inflammatory genes in human endothelial cells. CDC42 pathway was critically involved in senescence-associated inflammation and could be served as a therapeutic target for chronic inflammation in patients with inflammatory diseases (43). However, the directly relationship between CDC42 and AR had not been reported. PRDM10 was a member of the PRDM family, which has emerged as prime regulators of many types of tissue differentiation and disease pathogenesis (44-46). Studies demonstrated that PRDM family played critical roles in the development of inflammatory diseases. For example, PRDM1 genetic variants could be used to prognose, diagnose, and treat inflammatory disease (47). PRDM11 mutation was associated with inflammatory response in mice (48). However, the direct relationship between PRDM10 and AR were still not well understood.

Bioinformatics methods could help us identify significant genes and pathways related to the pathogenesis of AR. Steroid biosynthesis pathway and metabolic pathways might play an important role in the development of AR. Genes such as CDC42EP5, SLC39A11 and PRDM10 might be also associated with the pathogenesis of AR. However, further studies were still needed to confirm our results and explore the specific regulatory mechanism of these genes and pathways.

\section{Acknowledgements}

We would like to thank all the members of our group for their enthusiastic participation in this study.

\section{References}

1. Min YG: The pathophysiology, diagnosis and treatment of allergic rhinitis. Allergy Asthma Immunol Res 2: 65-76, 2010.

2. Meltzer EO, Nathan R, Derebery J, Stang PE, Campbell UB, Yeh WS, Corrao M and Stanford R: Sleep, quality of life, and productivity impact of nasal symptoms in the United States: Findings from the Burden of Rhinitis in America survey. Allergy Asthma Proc 30: 244-254, 2009.

3. Makino Y, Noguchi E, Takahashi N, Matsumoto Y, Kubo S, Yamada T, Imoto Y, Ito Y, Osawa Y, Shibasaki M, et al: Apolipoprotein A-IV is a candidate target molecule for the treatment of seasonal allergic rhinitis. J Allergy Clin Immunol 126: 1163-1169.e5, 2010.

4. Nathan RA: The burden of allergic rhinitis. Allergy Asthma Proc 28: 3-9, 2007.
5. Passalacqua G and Durham SR; Global Allergy and Asthma European Network: Allergic rhinitis and its impact on asthma update: Allergen immunotherapy. J Allergy Clin Immunol 119: 881-891, 2007.

6. Calderon MA, Alves B, Jacobson M, Hurwitz B, Sheikh A and Durham S: Allergen injection immunotherapy for seasonal allergic rhinitis. Cochrane Database Syst Rev 24: CD001936, 2007.

7. Andiappan AK, Wang de Y, Anantharaman R, Parate PN, Suri BK, Low HQ, Li Y, Zhao W, Castagnoli P, Liu J and Chew FT: Genome-wide association study for atopy and allergic rhinitis in a singapore chinese population. PLoS One 6: e19719, 2011.

8. Dávila I, Mullol J, Ferrer M, Bartra J, del Cuvillo A, Montoro J, Jáuregui I, Sastre J and Valero A: Genetic aspects of allergic rhinitis. J Investig Allergol Clin Immunol 19 (Suppl 1): S25-S31, 2009.

9. Hanuskova E and Plevkova J: The role of histamine $\mathrm{H} 4$ receptors as a potential targets in allergic rhinitis and asthma. Open J Mol Int Physiol 3: 6-14, 2013.

10. Bunyavanich S, Melen E, Wilk JB, Granada M, Soto-Quiros ME, Avila L, Lasky-Su J,Hunninghake GM, Wickman M,Pershagen G, et al: Thymic stromal lymphopoietin (TSLP) is associated with allergic rhinitis in children with asthma. Clin Mol Allergy 9: 1, 2011 .

11. Thompson JA, Tan J and Greene CS: Cross-platform normalization of microarray and RNA-seq data for machine learning applications. Peerj 4: e1621, 2016.

12. Diboun I, Wernisch L, Orengo CA and Koltzenburg M: Microarray analysis after RNA amplification can detect pronounced differences in gene expression using limma. BMC Genomics 7: 252, 2006.

13. Zhao Z, Guo AY, van den Oord EJ, Aliev F, Jia P, Edenberg HJ, Riley BP, Dick DM, Bettinger JC, Davies AG, et al: Multi-species data integration and gene ranking enrich significant results in an alcoholism genome-wide association study. BMC Genomics 13 (Suppl 8): S16, 2012.

14. Yang J, Yu H, Liu BH, Zhao Z, Liu L, Ma LX, Li YX and Li YY: DCGL v2.0: An R package for unveiling differential regulation from differential co-expression. PLoS One 8: e79729, 2013.

15. Yu H, Liu BH, Ye ZQ, Li C, Li YX and Li YY: Link-based quantitative methods to identify differentially coexpressed genes and gene pairs. BMC Bioinformatics 12: 315, 2011.

16. Szklarczyk D, Franceschini A, Kuhn M, Simonovic M, Roth A, Minguez P, Doerks T, Stark M, Muller J, Bork P, et al: The STRING database in 2011: Functional interaction networks of proteins, globally integrated and scored. Nucleic Acids Res 39 (Database Issue): D561-568, 2011.

17. Franceschini A, Szklarczyk D, Frankild S, Kuhn M, Simonovic M, Roth A, Lin J, Minguez P, Bork P, von Mering C and Jensen LJ: STRING v9.1: Protein-protein interaction networks, with increased coverage and integration. Nucleic Acids Res 41 (Database Issue): D808-D815, 2013.

18. Brehmer D: Endonasal phototherapy with Rhinolight for the treatment of allergic rhinitis. Expert Rev Med Devices 7: 21-26, 2014.

19. Zhu XL, Ai ZH, Wang J, Xu YL and Teng YC: Weighted gene co-expression network analysis in identification of endometrial cancer prognosis markers. Asian Pac J Cancer Prev 13: 4607-4611, 2012 .

20. Blood Pressure Lowering Treatment Trialists' Collaboration, Turnbull F, Neal B, Pfeffer M, Kostis J, Algert C, Woodward M, Chalmers J, Zanchetti A and MacMahon S: Blood pressuredependent and independent effects of agents that inhibit the renin-angiotensin system. J Hypertens 25: 951-958, 2007.

21. Bernstein KE, Ong FS, Blackwell WL, Shah KH, Giani JF, Gonzalez-Villalobos RA, Shen XZ, Fuchs S and Touyz RM: A modern understanding of the traditional and nontraditional biological functions of angiotensin-converting enzyme. Pharmacol Rev 65: 1-46, 2012.

22. Okwan-Duodu D, Datta V, Shen XZ, GoodridgeHS, BernsteinEA, Fuchs S, Liu GY and Bernstein KE: Angiotensin-converting enzyme overexpression in mouse myelomonocytic cells augments resistance to Listeria and methicillin-resistant Staphylococcus aureus. J Biol Chem 285: 39051-39060, 2010.

23. Song GG, Kim JH and Lee YH: Associations between the insertion/deletion polymorphism of the angiotensin-converting enzyme and susceptibility to aortic aneurysms: A meta-analysis. J Renin Angiotensin Aldosterone Syst 16: 211-218, 2015. 
24. Lin H, Lin D and Zheng CQ: Angiotensin-converting enzyme insertion/deletion polymorphism associated with allergic rhinitis susceptibility: Evidence from 1410 subjects. J Renin Angiotensin Aldosterone Syst 15: 593-600, 2013.

25. Evans RM: The steroid and thyroid hormone receptor superfamily. Science 240: 889-895, 1988 .

26. LaForce $\mathrm{C}$ : Use of nasal steroids in managing allergic rhinitis. J Allergy Clin Immunol 103: S388-S394, 1999.

27. Mygind N: Topical steroid treatment for allergic rhinitis and allied conditions. Clin Otolaryngol Allied Sci 7: 343-352, 1982.

28. Benson M, Strannegård IL, Strannegård O and Wennergren G Topical steroid treatment of allergic rhinitis decreases nasal fluid TH2 cytokines, eosinophils, eosinophil cationic protein and IgE but has no significant effect on IFN-gamma, IL-1beta, TNF-alpha, or neutrophils. J Allergy Clin Immunol 106: 307-312, 2000.

29. Proud D, Baumgarten CR, Naclerio RM and Ward PE: Kinin metabolism in human nasal secretions during experimentally induced allergic rhinitis. J Immunol 138: 428-434, 1987.

30. Ciprandi G, De Amici M, Tosca M and Fuchs D: Tryptophan metabolism in allergic rhinitis: The effect of pollen allergen exposure. Hum Immunol 71: 911-915, 2010.

31. Wjst M and Hyppönen E: Vitamin D serum levels and allergic rhinitis. Allergy 62: 1085-1086, 2007.

32. Shi J, Zhang Y, Qi S, Liu G, Dong X, Huang N, Li W, Chen H and Zhu B: Identification of potential crucial gene network related to seasonal allergic rhinitis using microarray data. Eur Arch Otorhinolaryngol 274: 231-237, 2017.

33. Hauswald B and Yarin YM: Acupuncture in allergic rhinitis: A mini-review. Allergo J Int 23: 115-119, 2014.

34. Yu Y, Wu A, Zhang Z, Yan G, Zhang F, Zhang L, Shen X, Hu R, Zhang Y, Zhang K and Wang F: Characterization of the GufA subfamily member SLC39A11/Zip11 as a zinc transporter. J Nutr Biochem 24: 1697-1708, 2013.

35. Bao S and Knoell DL: Zinc modulates airway epithelium susceptibility to death receptor-mediated apoptosis. Am J Physiol Lung Cell Mol Physiol 290: L433-L441, 2006.

36. Besecker B, Bao S, Bohacova B, Papp A, Sadee W and Knoell DL: The human zinc transporter SLC39A8 (Zip8) is critical in zinc-mediated cytoprotection in lung epithelia. Am J Physiol Lung Cell Mol Physiol 294: L1127-L1136, 2008.

37. Prasad AS: Clinical, immunological, anti-inflammatory and antioxidant roles of zinc. Exp Gerontol 43: 370-377, 2008.
38. AlKhyat THAT, Alturaihy SH and Ali ZA: The relationship between tumour necrosis factor -alpha and zinc/copper ratio in iraqi patients with allergic rhinitis. Glob J Sci Front Res 13, 2013.

39. Hirsch DS, Pirone DM and Burbelo PD: A new family of Cdc42 effector proteins, CEPs, function in fibroblast and epithelial cell shape changes. J Biol Chem 276: 875-883, 2001.

40. Hall A: Rho GTPases and the actin cytoskeleton. Science 279: 509-514, 1998

41. Wesselborg S, Bauer MK, Vogt M, Schmitz ML and Schulze-Osthoff K: Activation of transcription factor NF-kappaB and p38 mitogen-activated protein kinase is mediated by distinct and separate stress effector pathways. J Biol Chem 272: 12422-12429, 1997.

42. Puls A, Eliopoulos AG, Nobes CD, Bridges T, Young LS and Hall A: Activation of the small GTPase Cdc42 by the inflammatory cytokines TNF(alpha) and IL-1, and by the Epstein-Barr virus transforming protein LMP1. J Cell Sci 112: 2983-2992, 1999.

43. Ito TK, Yokoyama M, Yoshida Y, Nojima A, Kassai H, Oishi K, Okada S, Kinoshita D, Kobayashi Y, Fruttiger M, et al: A crucial role for CDC42 in senescence-associated inflammation and atherosclerosis. PLoS One 9: e102186, 2014.

44. Kim KC and Huang S: Histone methyltransferases in tumor suppression. Cancer Biol Ther 2: 491-499, 2003.

45. Huang S: Histone methyltransferases, diet nutrients and tumour suppressors. Nat Rev Cancer 2: 469-476, 2002.

46. Park JA, Kim TH, Lee B, Kwon E and Kim KC: Expression of PRDM10 in arthritic synovial derived tissues. Genes Genomics 35: 685-691, 2013.

47. Center CM: Methods of using prdm1 genetic variants to prognose, diagnose and treat inflammatory bowel disease, 2011.

48. Horsch M, Aguilar-Pimentel JA, Bönisch C, Côme C, Kolster-Fog C, Jensen KT, Lund AH, Lee I, Grossman LI, Sinkler C, et al: Cox4i2, Ifit2, and Prdm11 Mutant Mice: Effective selection of genes predisposing to an altered airway inflammatory response from a large compendium of mutant mouse lines. PLoS One 10: $\mathrm{e} 0134503,2015$.

This work is licensed under a Creative Commons Attribution-NonCommercial-NoDerivatives 4.0 International (CC BY-NC-ND 4.0) License. 